\title{
Linkage and Association Studies of Joint Morbidity from Rheumatoid Arthritis
}

\author{
JIN-YOUNG MIN, KYOUNG-BOK MIN, JOOHON SUNG, and SUNG-IL CHO
}

ABSTRACT. Objective. To investigate the relationship between genetic variations of rheumatoid arthritis (RA) susceptibility in terms of joint morbidity.

Methods. We used data from Genetic Analysis Workshop 15. The Illumina linkage panel IV included 5858 single-nucleotide polymorphisms (SNP), with 5744 SNP passing quality control filters. The phenotypic variables analyzed were the level of rheumatoid factor (RF) and score on the Joint Alignment and Motion (JAM) scale. We modified the scale, dividing by RF values relevant to disease severity. Linkage analysis for affected sibling pairs was done using the MERLIN program, and family-based association tests were carried out using PLINK and FBAT software.

Results. We found a high peak (LOD = 3.29; NPL Z = 4.07) near the HLA-DRB1 region on chromosome 6. The linkage at $6 \mathrm{p} 24$ at rs1410766 [LOD $=2.66$; nonparametric linkage (NPL) Z $=3.23$ ] was statistically significant. Two other regions also showed possible linkage peaks: chromosome $7 \mathrm{q} 30$ at rs322812 (LOD = 2.47; NPL Z = 3.39) and chromosome $15 \mathrm{p} 34$ at rs347117 (LOD = 1.95; NPL $Z=2.80$ ). For the family-based association study, 7 SNP related to clinical RA severity were detected.

Conclusion. Genetic variations may lead to an enhanced risk of joint damage and increased levels of RF. Further studies are needed to elucidate the roles of other genes involved in RA and to explore whether the clinical signs of RA are associated with particular genetic variations. (First Release Dec 23 2009; J Rheumatol 2010;37:291-5; doi:10.3899/jrheum.090526)

Key Indexing Terms:

LINKAGE STUDY

JOINT ALIGNMENT AND MOTION SCALE
ASSOCIATION STUDY RHEUMATOID ARTHRITIS
Rheumatoid arthritis (RA) is a chronic and progressive inflammatory disease occurring in the synovial membrane that can lead to joint deformity and cartilage destruction. Its characteristic feature is the production of autoantibodies ${ }^{1}$. Although there are still no established markers specific to RA, rheumatoid factor (RF), an autoantibody against the Fc portion of immunoglobulin $\mathrm{G}$, has modest specificity and has been used in classification criteria for RA by the American College of Rheumatology $y^{2,3}$.

RF production is the leading indicator of RA severity ${ }^{4}$. However, RF production can also be detected in healthy subjects and is sometimes associated with age. Further, other diseases and environmental exposures can play a role in trigger-

From the Institute of Health and Environment and the Department of Epidemiology, School of Public Health, Seoul National University, Seoul; and Department of Preventive Medicine, Ajou University School of Medicine, Suwon, South Korea.

Supported by the BK21 Program.

J-Y. Min, PhD, Institute of Health and Environment, Seoul National University; K-B. Min, MD, PhD, Department of Preventive Medicine, Ajou University School of Medicine; J. Sung, MD; S-I. Cho, MD, ScD, Department of Epidemiology, School of Public Health, Seoul National University.

Address correspondence to Dr. S-I. Cho, Department of Epidemiology, School of Public Health and Institute of Health and Environment, Seoul National University, Chongno-gu Yongeun-dong 28, Seoul, South Korea 110-460.E-mail: scho@snu.ac.kr

Accepted for publication September 29, 2009. ing RF production ${ }^{5}$. In a clinical setting, some patients with RA who have elevated RF levels appear to suffer more severe symptoms, such as progressive loss of joint motion and irreversible joint deformity, while other patients with RA, even those with high RF levels, appear to have relatively minor problems with joint function and disease severity ${ }^{4-6}$.

Although the progress and severity of RA can be measured by using objective biochemical examinations, given the current state of information, the disparity between these predictors and real symptoms is significant. While this disparity may be explained by phenotypic heterogeneity and individual variability, genetic variants could account for the majority of the difference.

We propose that a certain phenotype, manifested as clinical symptoms representing the degree of RA severity, could be influenced by genetic predisposition. These symptoms include joint deformity and loss of joint motion, 2 prominent features of RA responsible for the majority of unfavorable disease outcomes such as limited daily activity, reduced quality of life, and treatment compliance problems. We investigated genetic variations to RA susceptibility in relation to these symptoms of joint morbidity, using a linkage study and family-based association tests. Although it is unlikely that RF production is responsible for the joint destruction of RA, RF involves the formation of an immune complex that interacts with complement and effector cells to

Personal non-commercial use only. The Journal of Rheumatology Copyright (C) 2010. All rights reserved. 
damage the target cells, potentially promoting joint inflammation and immune dysregulation. Thus, the synovium may convert to lymphoid granulation tissue ${ }^{6}$. The measurement of joint morbidity used for this study was the joint alignment and motion (JAM) scale ${ }^{7,8}$. To compensate for the disparity between objective markers and clinical symptoms, we modified the scale by dividing JAM scores by RF values to obtain a ratio relevant to disease susceptibility. Patients with lower ratios presented less severe symptoms in terms of joint mobility and/or higher RF levels.

\section{MATERIALS AND METHODS}

Study population. We used the resources of the 15th Genetic Analysis Workshop database and the North American Rheumatoid Arthritis Consortium (NARAC) family collection. Families were recruited from 12 NARAC centers in the United States according to the following criteria: (1) at least 1 sibling had documented erosions on hand radiographs, and (2) at least 1 sibling had disease onset between age 18 and 60 years ${ }^{9}$. The dataset included 723 families with 1560 siblings with RA. Additionally, the analysis of RA affection status included data for patients with RA and 349 controls in the same dataset.

The Illumina linkage panel IV was used for analysis of genome-wide single-nucleotide polymorphisms (SNP) in all the families (Illumina, San Diego, CA, USA) ${ }^{10}$. The linkage panel contained 5858 SNP markers, and $98.1 \%$ of these markers passed quality control filters (minor allele frequency $>5 \%$, SNP call rate $>90 \%$, and Hardy-Weinberg equilibrium $\mathrm{p}$ value $>$ 0.01 ), giving a total yield of 5744 SNP. The results of the analysis of NARAC Caucasian families have been published ${ }^{11}$. Detailed information on the study subjects is available at the NARAC Website ${ }^{12}$.

We used RF levels and the JAM scale as phenotypic measures. In affected siblings, the mean values were $261.6 \mathrm{IU} / \mathrm{ml}$ (range 8-6920 IU/ml) for RF levels and 30.8 (range 0-118) on the JAM scale. RF is an IgM antibody directed against the $\mathrm{Fc}$ region of $\mathrm{IgG}$ and a commonly used serologic marker for the diagnosis of RA ${ }^{5}$. The JAM scale, as a measure of joint motility, represents the severity or limiting aspect of either motion or alignment ${ }^{7,8}$. It consists of a 5-point scale, with scores indicating the percentage of normal range of motion (ROM) and alignment: a score of 0 represents normal ROM and alignment; $1=0-5 \%$ decrease in ROM or malalignment; $2=6 \%-25 \%$ decrease in ROM or mild malalignment; $3=26 \%-75 \%$ decrease in ROM or joint subluxation; and $4=76 \%-100 \%$ decrease in ROM, joint fusion, or joint dislocation? ${ }^{7}$.

Statistical analysis. As a result of the joint susceptibility to RA, we calculated a JAM-RF ratio. This value was obtained by dividing the JAM scale score by RF level (JAM-RF ratio = JAM scale score/RF).

Linkage analysis for affected sibling pairs was done using the MERLIN program ${ }^{13}$ developed by Abecasis, et $a l^{14}$. From the linkage analysis, we obtained the nonparametric linkage (NPL) Z score and LOD score using the method of Kong and $\mathrm{Cox}^{15}$. In addition, we generated graphic plots along the entire length of each chromosome. Genetic map positions were based on the Rutgers Map Build 36. The empirical p value was estimated for the NPL Z and LOD scores from 10,000 replicates. In each simulation, markers with similar allele frequencies were also generated under the assumption of no linkage.

We performed the transmission disequilibrium test with PLINK software $^{16}$ and the family-based association test with FBAT software (-e option) ${ }^{17}$ using the additive genetic model.

\section{RESULTS}

Table 1 describes the structures of the 723 families included in our study. Most families had 2 affected sibling pairs $(\mathrm{N}=$ 637, 88\%), and 990 affected sibling pairs were included in
Table 1. Affected sibling pair families.
No. of families

No. of families with 2 affected sibling pairs

No. of families with 3 affected sibling pairs

No. of families with 4 affected sibling pairs

No. of families with 5 affected sibling pairs

No. of families with 7 affected sibling pairs

No. of affected siblings

No. of affected sibling pairs

No. of typed individuals
723

637

66

14

5

1

1560

990

1640 this analysis. A total of 1640 subjects, including family members and affected siblings, composed our study population.

Figure 1 displays the multipoint nonparametric LOD score of a genome-wide scan for the JAM-RF ratio over chromosomes 1-22. There was significant evidence of linkage to chromosome 6p24 at rs1410766 (LOD $=2.66$; NPL Z $=$ $3.23)$. The high peak $(\mathrm{LOD}=3.29$; NPL $Z=4.07)$ was also observed on chromosome 6 at approximately $5 \mathrm{cM}$ near rs197987 and rs451174. There were 2 suggestive regions, including chromosome $7 \mathrm{q} 30$ at $\mathrm{rs} 322812(\mathrm{LOD}=2.47$; NPL $\mathrm{Z}=3.39$ ) and chromosome $15 \mathrm{p} 34$ at rs 347117 (LOD = 1.95; NPL $Z=2.80$ ).

For chromosomes 6,7 , and 15 , we performed a complementary analysis for measurements related to RA (Figure 2). Four analyses were conducted on RA status, RF level, JAM score, and JAM-RF ratio. The linkage peak was dependent on RA diagnostic measurements, and the values differed largely among the measurements, while very similar peak regions were detected in chromosomes 6,7 , and 15 . The highest peak $(\mathrm{LOD}=9.83)$ for RA affection status was observed about $5 \mathrm{cM}$ from rs1402405 and rs412735 on chromosome 6, and the region was included in HLA-DRB1.

Table 2 shows the results of family-based association tests and corresponding LOD scores for the JAM-RF ratio. There were 7 SNP on chromosomes 6,7 , and 15 with a $\mathrm{p}$ value $<0.05$, but none of these SNP reached statistical significance following a Bonferroni correction. The SNP on chromosome 6 showed the strongest association. The highest LOD scores among these SNP were for rs2040587 (LOD $=1.76)$ and rs1009130 (LOD = 1.72), but these SNP were not highly significant in association tests $(\mathrm{p}<0.05)$.

\section{DISCUSSION}

We performed a quantitative trait linkage analysis and family-based association test for joint susceptibility to RA, as measured by the JAM-RF ratio, for affected sibling pairs. As expected, the JAM-RF ratio appears to be associated with the HLA-DRB1 region on chromosome 6 (LOD = 3.29; NPL $Z=4.07)$. This is consistent with reports that found strong evidence for linkage between RA and chromosome 6 , which contains the candidate gene HLA-DRB $1^{18-20}$. Cranney, et $a l^{21}$ demonstrated an association between the 


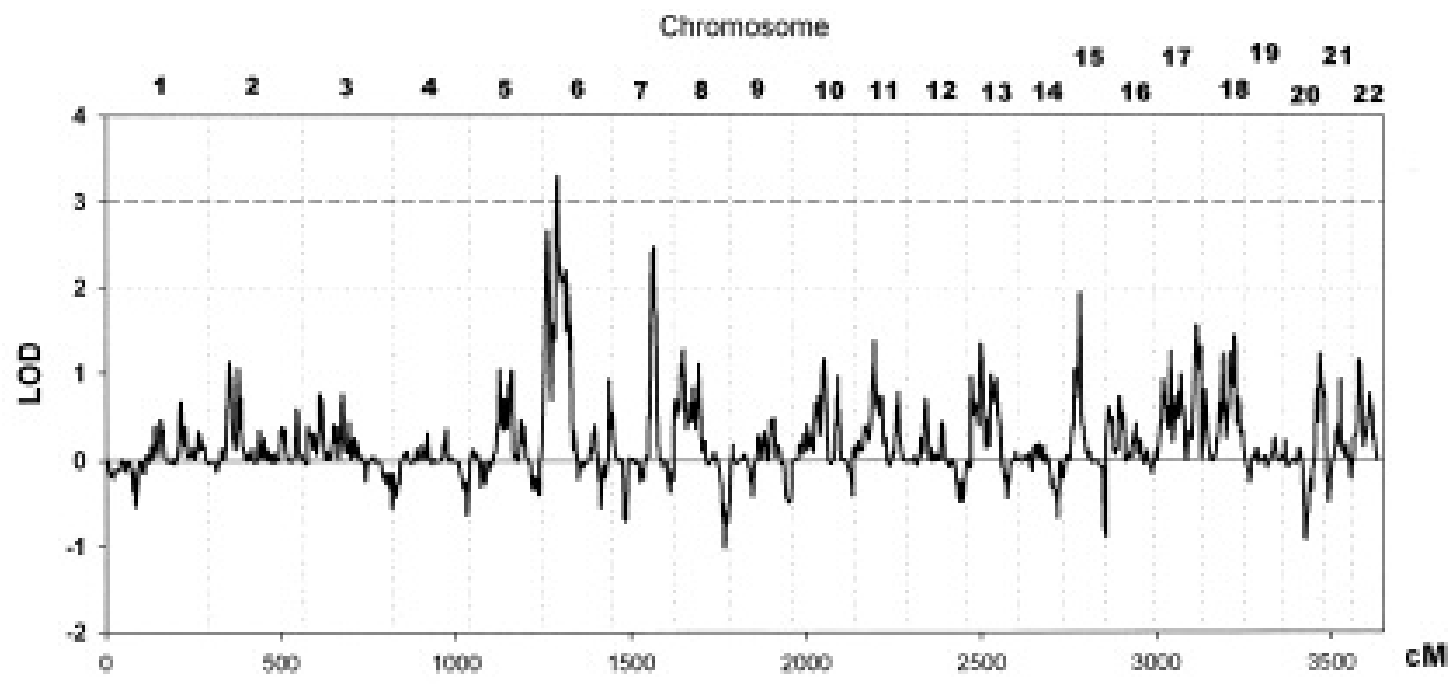

Figure 1. Multipoint nonparametric LOD scores for the rheumatoid factor-joint alignment and motion ratio.

HLA-DRB1 haplotype and disease severity as characterized by positive RF and higher degrees of joint deformity. Based on these results, we propose that HLA-DRB1 is not only the major region of genetic susceptibility to RA but is also a potential region for the joint deformity and loss of joint motion that occurs with RA.

The strongest finding was the significant evidence of linkage to region $6 \mathrm{p} 24(\mathrm{LOD}=2.66 ; \mathrm{NPLZ}=3.23)$. Among the many genes in the interval, an interesting candidate is bone morphogenetic protein 6 (BMP6) gene (MIM 112266), which is located less than $1 \mathrm{cM}$ from the linkage region of $6 \mathrm{p} 24$. The BMP are a family of secreted signaling molecules of the transforming growth factor- $\beta$ superfamily ${ }^{22}$. Several studies have reported a role for BMP6 in skeletal development and bone formation. Rickard, et a ${ }^{23}$ suggested that BMP6 production plays a role in mediating the effect of estrogen on development of bone and cartilage. Cheng, et $a l^{24}$ demonstrated a specific role for osteoblasts during mesenchymal stem cell differentiation. Further, work by Lories, et $a l^{25}$ supported the hypothesis that BMP6 could be a potent regulator of bone and cartilage repair in arthritis. Based on these results, we postulate that BMP6 may be responsible for helping to regulate joint motion and alignment during the progression of RA.

We observed 2 novel linkage regions on chromosomes $7 \mathrm{q} 30(\mathrm{LOD}=2.47 ; \mathrm{NPL} Z=3.39)$ and $15 \mathrm{p} 34(\mathrm{LOD}=1.95$; NPL $Z=2.80$ ) for JAM-RF ratio. No previous studies have reported linkage of these regions to RA. However, considering that the linkage analysis is dependent on phenotypic variants, in addition to other RA diagnostic measurements that display a similar pattern as shown in Figure 2, these 2 regions may contain candidate genes related to joint alignment and motion susceptibility to RA.

None of the findings in our study of potential linkage regions (chromosomes 6, 7, and 15) were significant after we applied a Bonferroni correction. There were 7 SNP with a $\mathrm{p}$ value $<0.05$, for which only 3 genes could be found: triadin (TRDN), Src homology 2 domain-containing family member 4 (SHC4), and hypothetical protein FLJ23834. TRDN is a protein that colocalizes with the ryanodine receptor on the sarcoplasmic reticulum in both skeletal muscle and cardiac muscle ${ }^{26}$. The triadin gene is a putative regulator of human muscular pathologies ${ }^{27,28}$. In contrast, the Shc family proteins serve as phosphotyrosine adaptor molecules in various receptor-mediated signaling pathways. The Shc family of docking proteins, $\mathrm{ShcD} / \mathrm{Shc} 4$, expressed in adult brain and skeletal muscle, may play a role in mediating downstream signaling of a muscle-specific kinase receptor $^{29}$. FLJ23834 is a putative calcium-dependent cell adhesion protein, and its function has not been established.

We could not confirm a causal link between these genes and clinical RA susceptibility as measured by the JAM-RF ratio. As a result, the association could be due to linkage disequilibrium between a marker and a phenotype. We also failed to detect statistically significant SNP following use of the Bonferroni correction. However, based on the plausible relationship between the novel genes and their potential roles in RA, our finding implies that the genes obtained from our family-based association tests may be responsible for the clinical susceptibility to RA as approximated by the JAM-RF ratio. In addition, other unknown genes could be present near these novel linkage regions.

Of interest is that no gene gave consistent results in both linkage analysis and genome-wide association tests. It is likely that the SNP used in the association analysis did not cover all of the genetic variations in the linkage regions because of the restricted number of SNP in the panel ${ }^{30}$. Another reason may be the lack of power of linkage analysis for complex traits, which could be influenced by multiple genetic, physical, and environmental features ${ }^{31}$. The Type I error of each test may also account for the discordant results between linkage and association analysis.

Personal non-commercial use only. The Journal of Rheumatology Copyright @ $\odot 2010$. All rights reserved. 

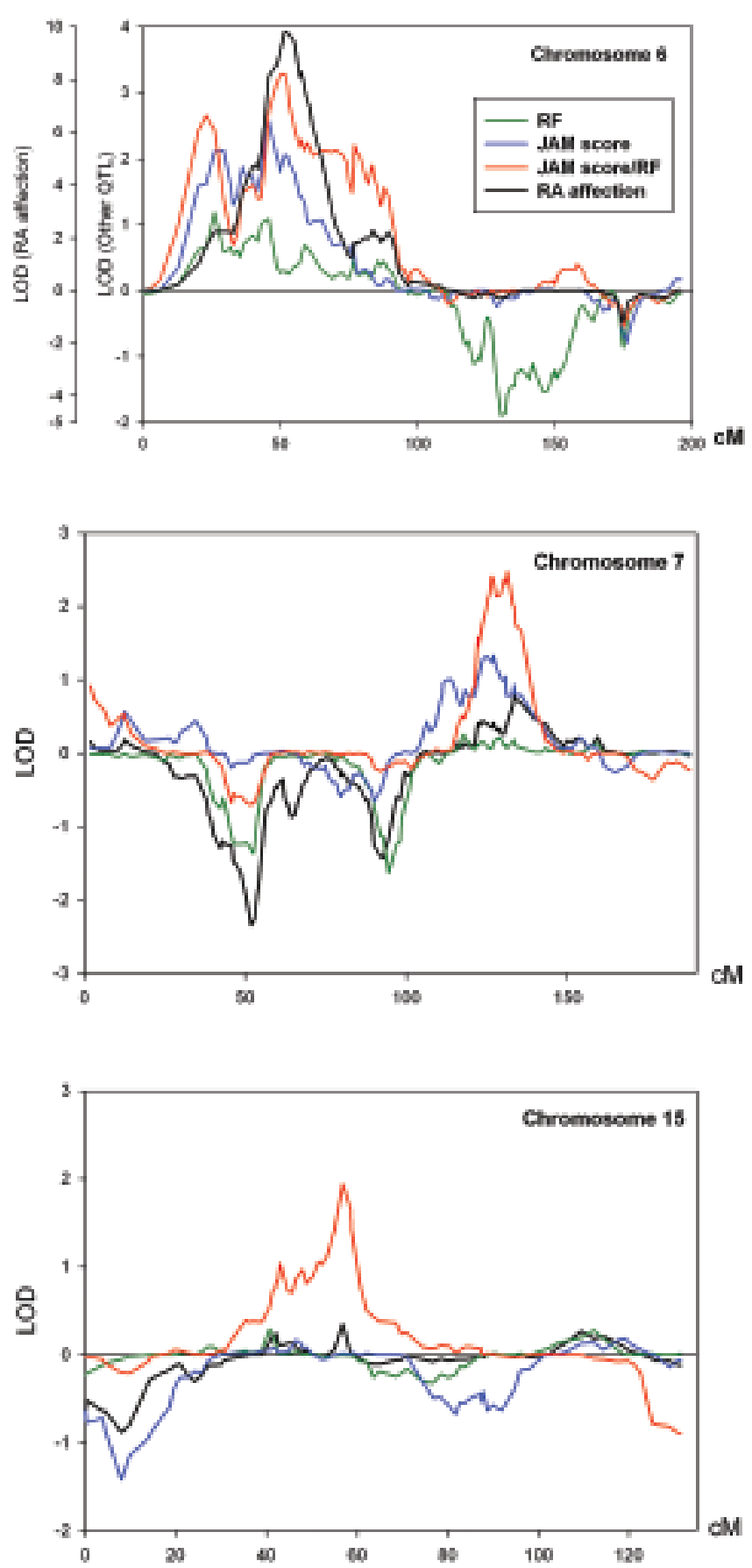

Figure 2. LOD scores on chromosomes 6 (A), 7 (B), and 15 (C) for measurements related to rheumatoid arthritis (RA). Analysis of RA affection status included data for patients with RA and 349 controls. Other analyses included data only for patients with RA. RF: rheumatoid factor; JAM: joint alignment and motion.

Research exploring genetic susceptibility to RA has increased, revealing significant evidence for linkage over wide chromosome regions to RA and the presence of RA-specific antibodies, such as RF and anti-citrullinated protein antibody $11,19,20,32,33$. The occurrence of RA has also been consistently associated with HLA-linked genes, as well as tumor necrosis factor, HLA-B, PADI4, CTLA4, TRAF1, STAT4, and PTPN22 $2^{34-38}$. Currently, genome projects have resulted not only in identification of genetic factors affecting health and disease but also in improved prediction and care for several diseases. Further, this progress has suggested new directions such as personalized medicine. Although major advances have been made in understanding its potential causes and in generating new therapeutic strategies, RA remains the most common autoimmune disease, with an unknown etiology and, at present, no cure. In light of these considerations, the association of specific gene variations with clinical susceptibility to RA could be used to formulate more effective diagnosis and treatment strategies. In addition, more aggressive treatments and intensive behavioral interventions would contribute to better diagnosis and management of RA.

We found that 2 loci show significant evidence of linkage at 6p21-24, and 2 other regions show suggestive linkage peaks at $7 \mathrm{q} 30$ and $15 \mathrm{p} 34$. Seven SNP related to joint susceptibility to RA were detected from family-based association tests. These results imply that genetic variations may affect the risk of joint damage and levels of RF. Further studies are needed to elucidate the roles of other genes involved in RA and to explore whether the clinical signs of RA are associated with particular genetic variations.

\section{REFERENCES}

1. Lee DM, Weinblatt ME. Rheumatoid arthritis. Lancet 2001;358:903-11.

2. Steiner G, Smolen J. Autoantibodies in rheumatoid arthritis and their clinical significance. Arthritis Res 2002;4:S1-5.

3. Arnett FC, Edworthy SM, Bloch DA, McShane DJ, Fries JF, Cooper NS, et al. The American Rheumatism Association 1987 revised criteria for the classification of rheumatoid arthritis. Arthritis Rheum 1988;31:315-24.

4. Cabral D, Katz JN, Weinblatt ME, Ting G, Avorn J, Solomon DH. Development and assessment of indicators of rheumatoid arthritis severity: results of a Delphi panel. Arthritis Rheum 2005;53:61-6.

5. Westwood OM, Nelson PN, Hay FC. Rheumatoid factors: what's new? Rheumatology 2006;45:379-85.

6. Harris ED Jr, Budd RC, Firestein GS, Genovese MC, Sergent JS, Ruddy S, et al. Kelley's textbook of rheumatology. 7th ed. Philadelphia: Elsevier Saunders; 2004:306.

7. Spiegel TM, Spiegel JS, Paulus HE. The joint alignment and motion scale: a simple measure of joint deformity in patients with rheumatoid arthritis. J Rheumatol 1987;14:887-92.

8. Parker JW, Harrell PB, Alarcón GS. The value of the joint alignment and motion scale in rheumatoid arthritis. J Rheumatol 1988;15:1212-5.

9. Jawaheer D, Seldin MF, Amos CI, Chen WV, Shigeta R, Monteiro $\mathrm{J}$, et al. A genomewide screen in multiplex rheumatoid arthritis families suggests genetic overlap with other autoimmune diseases. Am J Hum Genet 2001;68:927-36.

10. Illumina linkage panel IV. Illumina, San Diego, CA, USA. [Internet. Accessed Nov 3 2009.] Available from: http://www.illumina.com.

11. Amos CI, Chen WV, Lee A, Li W, Kern M, Lundsten R, et al. High-density SNP analysis of 642 Caucasian families with rheumatoid arthritis identifies two new linkage regions on $11 \mathrm{p} 12$ and 2q33. Genes Immun 2006; 7:277-86.

12. North American Rheumatoid Arthritis Consortium (NARAC). Manhasset, NY, USA. [Internet. Accessed October 30, 2009.] 
Table 2. Results of family-based association tests using the JAM-RF ratio and corresponding LOD scores.

\begin{tabular}{|c|c|c|c|c|c|c|c|c|c|}
\hline $\mathrm{Chr}$ & Marker & $\mathrm{cM}$ & Region & Gene & Allele & $\begin{array}{c}\text { Frequency of } \\
\text { Allele }\end{array}$ & $\begin{array}{c}\mathrm{FBAT}^{17} \\
\mathrm{p}\end{array}$ & $\begin{array}{c}\text { PLINK }^{16} \\
\mathrm{p}\end{array}$ & $\begin{array}{l}\text { MERLIN }^{13} \\
\text { LOD }\end{array}$ \\
\hline 6 & rs 1009130 & 84.22 & $6 \mathrm{p} 11.2$ & - & $\mathrm{A} / \mathrm{G}$ & 0.26 & 0.00435 & 0.00589 & 1.72 \\
\hline 6 & rs873460 & 126.96 & $6 \mathrm{q} 22.32$ & TRDN & $\mathrm{C} / \mathrm{T}$ & 0.39 & 0.00229 & 0.00155 & -0.02 \\
\hline 7 & rs41261 & 115.08 & $7 q 22.2$ & FLJ23834 & $\mathrm{C} / \mathrm{T}$ & 0.41 & 0.03774 & 0.01274 & 0.21 \\
\hline 7 & rs 2040587 & 123.59 & $7 \mathrm{q} 31.2$ & - & $\mathrm{C} / \mathrm{T}$ & 037 & 0.01944 & 0.01336 & 1.76 \\
\hline
\end{tabular}

JAM-RF: joint alignment and motion-rheumatoid factor; Chr: chromosome; TRDN: triadin; FLJ23834: hypothetical protein; SHC4: Scr homology 2 domain-containing family member 4 .

Available from: http://www.naracdata.org.

13. MERLIN program. Center for Statistical Genetics, University of Michigan, Ann Arbor, MI, USA. [Internet. Accessed October 30, 2009.] Available from:

http://www.sph.umich.edu/csg/abecasis/Merlin/.

14. Abecasis GR, Cherny SS, Cookson WO, Cardon LR.

Merlin - rapid analysis of dense genetic maps using sparse gene flow trees. Nat Genet 2002;30:97-101.

15. Kong A, Cox NJ. Allele-sharing models: LOD scores and accurate linkage tests. Am J Hum Genet 1997;61:1179-88.

16. PLINK software. Center for Human Genetic Research, Massachusetts General Hospital, Boston, MA, USA. [Internet. Accessed October 30, 2009.] Available from: http://pngu.mgh.harvard.edu/ purcell/plink/.

17. FBAT software. Program for Population Gentics, Harvard School of Public Health, Cambridge, MA, USA. [Internet. Accessed October 30, 2009.] Available from: http://www.biostat.harvard.edu/ fbat/fbat.htm.

18. Fisher SA, Lanchbury JS, Lewis CM. Meta-analysis of four rheumatoid arthritis genome-wide linkage studies: confirmation of a susceptibility locus on chromosome 16 . Arthritis Rheum 2003;48:1200-6.

19. John S, Amos C, Shephard N, Chen W, Butterworth A, Etzel C, et al. Linkage analysis of rheumatoid arthritis in US and UK families reveals interactions between HLA-DRB1 and loci on chromosomes 6q and 16p. Arthritis Rheum 2006;54:1482-90.

20. Choi SJ, Rho YH, Ji JD, Song GG, Lee YH. Genome scan meta-analysis of rheumatoid arthritis. Rheumatology 2006;45:166-70.

21. Cranney A, Goldstein R, Pham B, Newkirk MM, Karsh J. A measure of limited joint motion and deformity correlates with HLA-DRB1 and DQB1 alleles in patients with rheumatoid arthritis. Ann Rheum Dis 1999;58:703-8.

22. Kessler E, Takahara K, Biniaminov L, Brusel M, Greenspan DS. Bone morphogenetic protein-1: the type I procollagen C-proteinase. Science 1996;271:360-2.

23. Rickard DJ, Sullivan TA, Shenker BJ, Leboy PS, Kazhdan I. Induction of rapid osteoblast differentiation in rat bone marrow stromal cell cultures by dexamethasone and BMP-2. Dev Biol 1994;161:218-28.

24. Cheng H, Jiang W, Phillips FM, Haydon RC, Peng Y, Zhou L, et al. Osteogenic activity of the fourteen types of human bone morphogenetic proteins (BMPs). J Bone Joint Surg Am 2003;85:1544-52.

25. Lories RJ, Derese I, Ceuppens JL, Luyten FP. Bone morphogenetic proteins 2 and 6 , expressed in arthritic synovium, are regulated by proinflammatory cytokines and differentially modulate fibroblast-like synoviocyte apoptosis. Arthritis Rheum 2003;48:2807-18.

26. Peng M, Fan H, Kirley TL, Caswell AH, Schwartz A. Structural diversity of triadin in skeletal muscle and evidence of its existence in heart. FEBS Lett 1994;348:17-20.

27. Taske NL, Eyre HJ, O'Brien RO, Sutherland GR, Denborough MA, Foster PS. Molecular cloning of the cDNA encoding human skeletal muscle triadin and its localisation to chromosome 6q22-6q23. Eur J Biochem 1995;233:258-65.

28. Thevenon D, Smida-Rezgui S, Chevessier F, Groh S, Henry-Berger J, Beatriz Romero N, et al. Human skeletal muscle triadin: gene organization and cloning of the major isoform, Trisk 51. Biochem Biophys Res Commun 2003;303:669-75.

29. Jones N, Hardy WR, Friese MB, Jorgensen C, Smith MJ, Woody $\mathrm{NM}$, et al. Analysis of a Shc family adaptor protein, $\mathrm{ShcD} / \mathrm{Shc} 4$, that associates with muscle-specific kinase. Mol Cell Biol 2007;27:4759-73.

30. Carlson CS, Eberle MA, Rieder MJ, Yi Q, Kruglyak L, Nickerson DA. Selecting a maximally informative set of single-nucleotide polymorphisms for association analyses using linkage disequilibrium. Am J Hum Genet 2004;74:106-120.

31. Zhu X, Cooper R, Kan D, Cao G, Wu X. A genome-wide linkage and association study using COGA data. BMC Genet 2005;6:S128.

32. Osorio Y Fortéa J, Bukulmez H, Petit-Teixeira E, Michou L, Pierlot C, Cailleau-Moindrault S, et al. Dense genome-wide linkage analysis of rheumatoid arthritis, including covariates. Arthritis Rheum 2004;50:2757-65.

33. Criswell LA, Chen WV, Jawaheer D, Lum RF, Wener MH, Gu X, et al. Dissecting the heterogeneity of rheumatoid arthritis through linkage analysis of quantitative traits. Arthritis Rheum 2007;56:58-68.

34. Khanna D, Wu H, Park G, Gersuk V, Gold RH, Nepom GT, et al; Western Consortium of Practicing Rheumatologists. Association of tumor necrosis factor alpha polymorphism, but not the shared epitope, with increased radiographic progression in a seropositive rheumatoid arthritis inception cohort. Arthritis Rheum 2006;54:1105-16.

35. Harney SM, Meisel C, Sims AM, Woon PY, Wordsworth BP, Brown MA. Genetic and genomic studies of PADI4 in rheumatoid arthritis. Rheumatology 2005;44:869-72.

36. Yanagawa T, Gomi K, Nakao EI, Inada S. CTLA-4 gene polymorphism in Japanese patients with rheumatoid arthritis. J Rheumatol 2000;27:2740-2.

37. Zervou MI, Sidiropoulos P, Petraki E, Vazgiourakis V, Krasoudaki E, Raptopoulou A, et al. Association of a TRAF1 and a STAT4 gene polymorphism with increased risk for rheumatoid arthritis in a genetically homogeneous population. Hum Immunol 2008;69:567-71.

38. Begovich AB, Carlton VE, Honigberg LA, Schrodi SJ, Chokkalingam AP, Alexander $\mathrm{HC}$, et al. A missense single-nucleotide polymorphism in a gene encoding a protein tyrosine phosphatase (PTPN22) is associated with rheumatoid arthritis. Am J Hum Genet 2004;75:330-7. 